\title{
Minimum Weight Independent Neighborhood Sets of Interval Graphs
}

\author{
Dr. C.V.Sulochana
}

Lecturer, RSVP, Tirupati, AP, India.

\section{Prof. B. Maheswari}

S.P. Women's University, Tirupati, AP, India.

\begin{abstract}
The subject of graph theoretic algorithms is as old as graph theory itself, which is usually regarded as having started with Euler's famous solution of the problem of the seven bridges of Konigsberg.

Over the last three decades there has been substantial increase in the study of algorithmic graph theory. Many projects involving graphs even in pure graph theory itself, involve algorithms.

Most of the real life problems when transformed into graph problems exhibit some special properties. This has given rise to special classes of graphs such as interval graphs, permutation graphs, circle graphs, circular-arc graphs, etc.

In this paper, we present a method for finding a minimum weight independent neighborhood set (MWINS) of an interval graph by using a directed network.
\end{abstract}

Keywords: Neighborhood Set, Independent Set, Weighted Graph

\section{INTRODUCTION}

Consider a graph $\mathrm{G}(\mathrm{V}, \mathrm{E})$. This graph is said to be an interval graph if there is a one-one correspondence between the vertex set $\mathrm{V}$ and the interval family I in such way that two vertices of $\mathrm{G}$ are joined by an edge in $E$ if and only if their corresponding intervals in I intersect. i.e if $i=\left[a_{i}, b_{i}\right]$ and $j=\left[a_{j}, b_{j}\right]$ then $i$ and $j$ intersect means $a_{j}<b_{i}$ or $a_{i}<b_{j}$.

The neighborhood of a vertex $\mathrm{v}$ in $\mathrm{G}$ is defined as the set of vertices adjacent with $\mathrm{v}$ (including $\mathrm{v}$ ) and is denoted by ndb [v]. A subset $\mathrm{S}$ of $\mathrm{V}$ in $\mathrm{G}$ is called a neighborhood set of $\mathrm{G}$ if $\mathrm{G}=\underset{v \in S}{\cup}\langle\operatorname{nbd}[\mathrm{v}]\rangle$ where $<\operatorname{nbd}[\mathrm{v}]>$ is the vertex induced sub graph of G. A neighborhood set with minimum cardinality is called a minimum neighborhood set and the cardinality of such a set is defined as the neighborhood number of $\mathrm{G}$.

Each vertex of $\mathrm{G}$ is assigned a real number called its weight. The weights are assigned arbitrarily to the vertices of $\mathrm{G}$. The weight of a set $\mathrm{S}$ is the sum of the weights on its elements. A neighborhood set of $\mathrm{G}$ with a minimum weight is called a minimum weight neighborhood set. If the neighborhood set with minimum weight is independent, then it is called a minimum weight independent neighborhood set.

An algorithm for finding a minimum weight independent neighborhood set and the necessary concepts for execution of the algorithm are given as follows.

Let min (i) denote the smallest interval in nbd [i]. If $B$ is any subset of $V$ then low (B) is defined as the interval with minimum weight in B. Define NI [i] $=j$ if $b_{i}<a_{j}$ and there doesn't exist an interval $k$ such that $b_{i}<a_{k}<a_{j}$. If there is no such $j$ then define NI [i] $=$ null.

Define Next (i) = low $\{\operatorname{nbd}[\min (\mathrm{NI}[\mathrm{i}])] \backslash \operatorname{nbd}[\mathrm{i}]\}$ and

Next $(\mathrm{i})=$ null if $\mathrm{NI}[\mathrm{i}]=$ null

First we augment I with two dummy intervals say, $I_{0}$ and $I_{n+1}$ where $I_{o}=\left[a_{0}, b_{o}\right]$ and $I_{n+1}=\left[a_{n+1}, b_{n+1}\right]$ such that 


$$
\text { bo }<\min _{1 \leq k \leq n}\left\{a_{k}\right\} \text { and } \mathrm{a}_{\mathrm{n}+1}>\max _{1 \leq k \leq n}\left\{b_{k}\right\}
$$

Let $I_{1}=I \cup\left\{I_{0}, I_{n+1}\right\}$. As in $I$ the intervals in $I_{1}$ are also indexed by increasing order of their right end points, namely $b_{0}<b_{1}<\ldots . .<b_{n+1}$.

For convenience, we denote $I_{0}$ by 0 and $I_{n+1}$ by $n+1$.

We now construct a directed network D (N, L) associated with G. For its nodes we take those intervals in $\mathrm{I}_{1}$ which are not properly contained within other intervals. Because if there is an interval $\mathrm{j}$ which contains another interval $i$, then the minimum weight independent neighborhood set containing $\mathrm{i}$ can be changed to $\{$ MWINS $\backslash \mathrm{i}\} \cup\{\mathrm{j}\}$. $\mathrm{L}_{2}=\phi$.

The lines in $\mathrm{L}$ are partitioned into two disjoint sets $\mathrm{L}_{1}$ and $\mathrm{L}_{2}$ such that $\mathrm{L}=\mathrm{L}_{1} \cup \mathrm{L}_{2}$ and $\mathrm{L}_{1} \cap$

The lines in $\mathrm{L}_{1}$ are defined as follows. For $\mathrm{j} \in \mathrm{D}(\mathrm{N}, \mathrm{L})$ there is a directed line $(0, \mathrm{j})$ between 0 and $\mathrm{j}$ that belongs to $L_{1}$ if and only if there is no interval $h$ such that $b_{0}<a_{h}<b_{h}<a_{j}$. Similarly there is a directed line $(j, n+1)$ between $j$ and $n+1$ that belongs to $L_{1}$ if and only if there is no interval $h$ such that $b_{j}<a_{h}<b_{h}<a_{n+1}$. This gives scope to join the intervals 0 and $n+1$ to other intervals in I and all such joined directed lines belong to $\mathrm{L}_{1}$.

Next the lines in $L_{2}$ are defined as follows. For $i, j \in D(N, L)$, there is a directed line (i, j) between $i$ and $j$ that belongs to $L_{2}$ if and only $j=\operatorname{Next}(i)$.

Now the following algorithm finds a minimum weight independent neighborhood set of an interval graph.

\section{ALGORITHM: MWINS-IG}

Input : Interval family $\mathrm{I}=\{1,2 \ldots \ldots \mathrm{n}\}$.

Output : Minimum weight independent neighborhood set in G.

Step 1 : Construct a directed network D (N,L).

Step 2 : Find a minimum weight directed path $\mathrm{P}$ from node 0 to $\mathrm{n}+1$

in $\mathrm{D}(\mathrm{N}, \mathrm{L})$.

Step 3 : The nodes of $\mathrm{P}$ forms a minimum weight independent neighborhood set.

Step 4 : End.

\section{Illustration}

Let us illustrate the construction of a directed network and the method of finding a minimum weight independent neighborhood set.

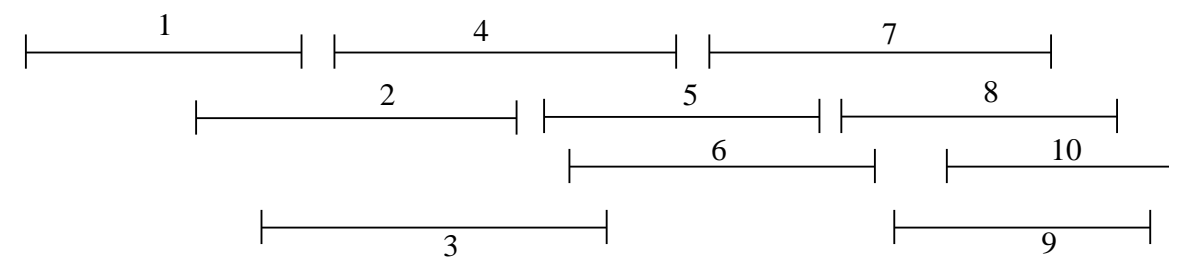

Fig. 1. Interval Family

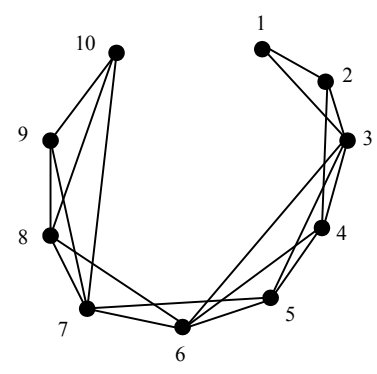

Fig.2. Interval Graph 


$$
\begin{aligned}
& \begin{array}{lllll}
\text { Let } & \text { Wt }(1)=4 & \text { Wt }(2)=6 & \text { Wt }(3)=8 & \text { Wt (4) }=1
\end{array} \\
& \text { Wt }(5)=6 \quad \text { Wt }(6)=2 \quad \text { Wt }(7)=3 \quad \text { Wt }(8)=4 \\
& \mathrm{Wt}(9)=2 \quad \mathrm{Wt}(10)=5 \\
& \operatorname{nbd}[1]=\{1,2,3\} \\
& \min (1)=1 \\
& \text { NI [1] }=4 \\
& \operatorname{nbd}[2]=\{1,2,3,4\} \\
& \min (2)=1 \\
& \mathrm{NI}[2]=5 \\
& \operatorname{nbd}[3]=\{1,2,3,4,5,6\} \quad \min (3)=1 \\
& \operatorname{nbd}[4]=\{2,3,4,5,6\} \\
& \min (4)=2 \\
& \text { NI [3] }=7 \\
& \operatorname{nbd}[5]=\{3,4,5,6,7\} \\
& \min (5)=3 \\
& \text { NI [4] }=7 \\
& \operatorname{nbd}[6]=\{3,4,5,6,7,8\} \quad \min (6)=3 \\
& \operatorname{nbd}[7]=\{5,6,7,8,9,10\} \quad \min (7)=5 \\
& \operatorname{nbd}[8]=\{6,7,8,9,10\} \quad \min (8)=6 \\
& \operatorname{nbd}[9]=\{7,8,9,10\} \\
& \min (9)=7 \\
& \text { NI [5] }=8 \\
& \operatorname{nbd}[10]=\{7,8,9,10\} \\
& \min (10)=7 \\
& \text { NI [6] }=9 \\
& \text { NI [7] = null } \\
& \text { NI [8] = null } \\
& \text { NI [9] = null } \\
& \mathrm{NI}[10]=\text { null } \\
& =\quad \operatorname{low}\{\operatorname{nbd}[\min (\mathrm{NI}[1])] \backslash \operatorname{nbd}[1]\} \\
& =\quad \operatorname{low}\{\operatorname{nbd}[\min (4)] \backslash \operatorname{nbd}[1]\} \\
& =\quad \operatorname{low}\{\operatorname{nbd}[2] \backslash \operatorname{nbd}[1]\}=\operatorname{low}\{\{1,2,3,4\} \backslash\{1,2,3\}\} \\
& =\operatorname{low}\{4\}=4
\end{aligned}
$$

$\operatorname{Next}(2) \quad=\quad$ low $\{\operatorname{nbd}[\min (\mathrm{NI}[2])] \backslash \operatorname{nbd}[2]\}$

$=\quad \operatorname{low}\{\operatorname{nbd}[\min (5)] \backslash \operatorname{nbd}[2]\}$

$=\operatorname{low}\{\operatorname{nbd}[3] \backslash \operatorname{nbd}[2]\}=\operatorname{low}\{\{1,2,3,4,5,6\} \backslash\{1,2,3,4\}\}$

$$
=\operatorname{low}\{5,6\}=6
$$

$\operatorname{Next}(3) \quad=\quad \operatorname{low}\{\operatorname{nbd}[\min (\mathrm{NI}[3])] \backslash \operatorname{nbd}[3]\}$

$=\quad \operatorname{low}\{\operatorname{nbd}[\min (7)] \backslash \operatorname{nbd}[3]\}$

$=\quad \operatorname{low}\{\operatorname{nbd}[5] \backslash \operatorname{nbd}[3]\}=\operatorname{low}\{\{3,4,5,6,7\} \backslash\{1,2,3,4,5,6\}\}$

$$
=\operatorname{low}\{7\}=7
$$

Next $(4) \quad=\quad \operatorname{low}\{\operatorname{nbd}[\min (\mathrm{NI}[4])] \backslash \operatorname{nbd}[4]\}$

$=\quad \operatorname{low}\{\operatorname{nbd}[\min (7)] \backslash \operatorname{nbd}[4]\}$

$=\quad \operatorname{low}\{\operatorname{nbd}[5] \backslash \operatorname{nbd}[4]\}=$ low $\{\{3,4,5,6,7\} \backslash\{2,3,4,5,6\}\}$

$$
=\operatorname{low}\{7\}=7
$$


Dr. C.V. Sulochana \& Prof. B. Maheswari

\begin{tabular}{rlrl}
\hline Next (5) & $=$ & $\operatorname{low}\{\operatorname{nbd}[\min (\mathrm{NI}[5])] \backslash \operatorname{nbd}[5]\}$ \\
& $=$ & & $\operatorname{low}\{\operatorname{nbd}[\min (8)] \backslash \operatorname{nbd}[5]\}$ \\
& $=$ & & $\operatorname{low}\{\operatorname{nbd}[6] \backslash \operatorname{nbd}[5]\}=\operatorname{low}\{\{3,4,5,6,7,8\} \backslash\{3,4,5,6,7\}\}$ \\
Next (6) & $=$ & $\operatorname{low}\{\operatorname{nbd}[\min (\mathrm{NI}[6])] \backslash \operatorname{nbd}[6]\}$ \\
& $=$ & $\operatorname{low}\{\operatorname{nbd}[\min (9)] \backslash \operatorname{nbd}[6]\}$ \\
& $=$ & $\operatorname{low}\{\operatorname{nbd}[7] \backslash \operatorname{nbd}[6]\}=\operatorname{low}\{\{5,6,7,8,9,10\} /\{3,4,5,6,7,8\}\}$ \\
& & $=\operatorname{low}\{9,10\}=9$
\end{tabular}

$\begin{array}{lll}\text { Next }(7) & = & \text { null, since NI [7] }=\text { null. } \\ \text { Next (8) } & = & \text { null, since NI [8] = null. } \\ \text { Next (9) } & = & \text { null, since NI [9] = null. } \\ \text { Next (10) } & = & \text { null, since NI [10] = null. }\end{array}$

Now dummy intervals 0 and $n+1$ are augmented to I.

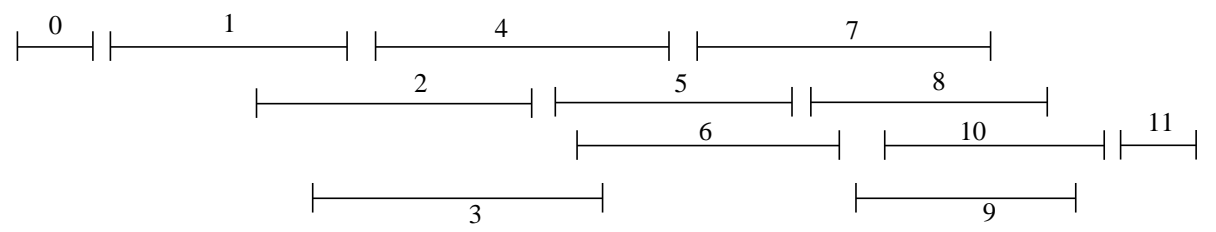

Fig. 3. Interval Family, $I_{1}=I \cup\{0, n+1\}$

Directed Network D (N, L) is constructed as follows.

$\mathrm{N}=\{0,1,2,3,4,5,6,7,8,9,10,11\}$

$\mathrm{L}=\mathrm{L}_{1} \cup \mathrm{L}_{2}$

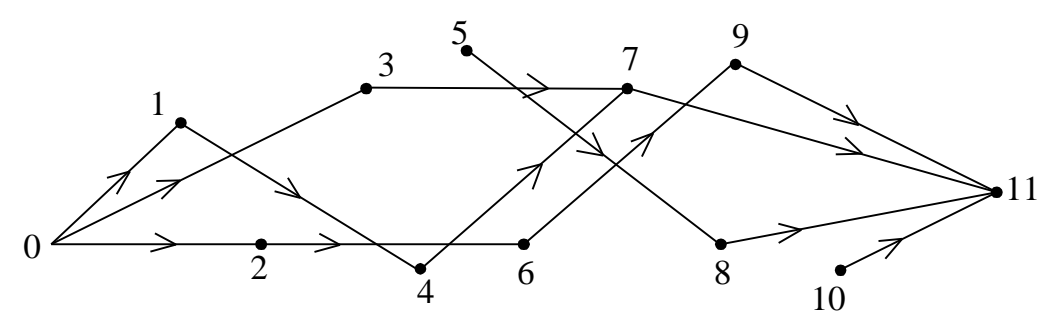

Fig. 4. The directed network $D(N, L)$

In the $\mathrm{D}(\mathrm{N}, \mathrm{L})$ of the above example, observe that directed paths from node 0 to node 11 are $\mathrm{P}_{1}=(0$, $3,7,11), P_{2}=(0,1,4,7,11)$ and $P_{3}=(0,2,6,9,11)$. The weights on these paths are respectively 11 , 8 , and 10 . Hence $(0,1,4,7,11)$ is a minimum weight directed path which corresponds to a minimum weight independent neighborhood set namely $\{1,4,7\}$ of the interval graph with weight

\section{RESULTS}

Lemma 1: if $\mathrm{i}$ and $\mathrm{k}$ are any two intersecting intervals and $\mathrm{j}$ is any interval such that $\mathrm{i}<\mathrm{j}<\mathrm{k}$ then $\mathrm{j}$ intersects $\mathrm{k}$.

Proof : Since the intervals are labeled in the increasing order of their right endpoints, $i<j<k$ implies that $b_{i}<b_{j}<b_{k}$. Now $i$ intersects $k$ implies that

$a_{k}<b_{i}$. Hence $a_{k}<b_{i}<b_{j}<b_{k}$. This implies that $j$ intersects $k$. 
Lemma 2: For any directed line $(0, \mathrm{j}) \in \mathrm{L}_{1}$ where $\mathrm{j}$ is any interval of $\mathrm{I}$, the intervals between 0 and $\mathrm{j}$ belong to nbd [j].

Proof : Let $(0, j) \in L_{1}$. By the definition of the lines in $L_{1}$, there is no interval $h$ such that $b_{o}<a_{h}<b_{h}$ $<\mathrm{a}_{\mathrm{j}}$. Therefore the intervals between 0 and $\mathrm{j}$ belong to $\mathrm{nbd}[\mathrm{j}]$.

The proof of the following lemma follows on similar lines to that of Lemma 2.

Lemma 3 : For any directed line $(j, n+1) \in L_{1}$ where $j$ is any interval of $I$, the intervals between $j$ and $\mathrm{n}+1$ belong to nbd [j].

Thus it is clear by lemmas 2 and 3 that if there is a directed line $(i, j) \in L_{1}$ then the intervals between $i$ and $j$ are adjacent with $i$ or $j$.

Lemma 4: For any directed line $(i, j) \in L_{2}$ the intervals between $i$ and $\mathrm{j}$ belong to nbd [i] or nbd [j].

Proof : Let $(i, j) \in L_{2}$. Then $j=$ Next (i). Let $h$ be any interval between $i$ and $j$. Then the following cases arise.

Case1: Suppose h intersects i. In such case h $\in$ nbd [i].

Case 2: Suppose $\mathrm{h}$ intersects $\min (\mathrm{NI}[\mathrm{i}])$ and does not intersect i. Then $\mathrm{h} \in \operatorname{nbd}[\min (\mathrm{NI}[\mathrm{i}])]$ and $\mathrm{h} \notin \mathrm{nbd}[\mathrm{i}]$. So $\mathrm{h} \in\{\operatorname{nbd}[\min (\mathrm{NI}(\mathrm{i}))] \backslash \mathrm{nbd}[\mathrm{i}]\}$. Since $\mathrm{j}$ is the element with minimum weight in nbd[min (NI [i])] and the intervals are labeled in the increasing order of their right endpoints,

it follows that $\mathrm{h}$ must intersect $\mathrm{j}$. That is $\mathrm{h} \in \mathrm{nbd}[\mathrm{j}]$.

Case 3 : Assume that $\mathrm{h}$ does not intersect neither i nor $\min (\mathrm{NI}[\mathrm{i}])$. Then either $\mathrm{i}<\mathrm{h}<\min (\mathrm{NI}[\mathrm{i}])$ or $\min (\mathrm{NI}[\mathrm{i}])<\mathrm{h}<\operatorname{Next}(\mathrm{i})$.

Suppose $\mathrm{i}$ and min (NI[i]) intersect. Then by Lemma 1, h and min (NI[i]) intersect. Suppose min (NI[i]) and Next (i) intersect. Then again by Lemma 1, h must interesect Next (i). Hence our assumption that $\mathrm{h}$ does not intersect neither i nor $\min (\mathrm{NI}[\mathrm{i}])$ does not arise.

Case 4 : Suppose $h$ intersects j. Then clearly $h \in$ nbd [j].

Thus for all possibilities, the intervals between $\mathrm{i}$ and $\mathrm{j}$ belong to $\mathrm{nbd}[\mathrm{i}]$ or $\mathrm{nbd}[\mathrm{j}]$.

Lemma 5 : If $i, j$ are any two intervals in $I$ such that $j=\operatorname{Next}(i)$, then $i$ and $j$ are non-adjacent.

Proof : By the definition of Next (i), the proof follows immediately.

Theorem 1: If $\mathrm{P}$ is a directed path between the nodes 0 to $n+1$ in $\mathrm{D}(\mathrm{N}, \mathrm{L})$ then the vertices in $\mathrm{P}$ other than 0 and $\mathrm{n}+1$ correspond to an independent neighbourhood set of an interval graph.

Proof: Let $P$ be a shortest directed path from node 0 to $n+1$ in $D$. Define $S=\{i$ : node $i$ appears in $P, i \neq 0, i \neq n+1\}$. For each directed line $(i, j)$ in $P$, by Lemma 4 it follows that all intermediate intervals $i+1, i+2 \ldots j-1$ between $i$ and $j$ belong to nbd [i] $\cup$ nbd [j]. Hence all intermediate intervals between the intervals in $\mathrm{S}$ belong to $\underset{i, j \in S}{\cup}<\operatorname{nbd}[\mathrm{i}] \cup \operatorname{nbd}[\mathrm{j}]\rangle$. Since the intervals in $\mathrm{S}$ correspond to the nodes in path $\mathrm{P}$, the intervals in between 0 and the first interval in $\mathrm{S}$ as well as the intervals in between the last interval in $\mathrm{S}$ and $\mathrm{n}+1$ also belong to $\underset{i \in S}{\cup}<n b d[i]>$, by Lemmas 2 and 3. Thus all the vertices in $\mathrm{G}$ are exhausted by the vertices in the neighbourhoods of the nodes in $S$. That is $\mathrm{V}(\mathrm{G})=\cup_{i \in S} n b d[i]$. But by Lemma $5,<\{\mathrm{i} \ldots \mathrm{j}\}>\subseteq<\operatorname{nbd}[\mathrm{i}] \cup \operatorname{nbd}[\mathrm{j}]>$ where $\mathrm{i}, \mathrm{j} \in \mathrm{S}$. Therefore $\underset{i, j \in S}{\cup}<\{\mathrm{i} \ldots \mathrm{j}\}>\subseteq \underset{i, j \in S}{\cup}<n b d[i] \cup n b d[j]>$. Since $\mathrm{V}(\mathrm{G})=\cup_{i \in S}<n b d[i]>$, it follows that $\mathrm{G}=$ $\cup_{i \in S}<n b d[i]>$. Thus $\mathrm{S}$ is a neighborhood set of G. By Lemma 6 the nodes in $\mathrm{S}$ are non-adjacent. Therefore $\mathrm{S}$ forms an independent neighborhood set of $\mathrm{G}$. 
Theorem 2 : A minimum weight directed path $\mathrm{P}$ in $\mathrm{D}(\mathrm{N}, \mathrm{L})$ corresponds to a minimum weight independent neighborhood set of an interval graph.

Proof : Proof follows immediately by Theorem 1.

\section{CONCLUSION}

In this article we found an algorithm to find minimum weight independent neighbourhood set of interval graphs using a directed network. Also we proved some results on directed network. Finally we conclude that the minimum weight directed path $P$ in $D(N, N)$ corresponds to a minimum weight independent neighbourhood set of interval graph.

\section{REFERENCES}

[1] Bhaskarudu, P. - A study of some types of domination in graphs, Ph.D. Thesis, Sri Venkateswara University, India, (2002).

[2] Bondy and Murthy - Graph theory with applications, Macmillan (1976).

[3] Kattimani, M.B. - Some new contributions to Graph theory, Ph.D. thesis, Gulbarga University, India, (2000).

[4] Lakshmi Naidu, Y. - Some studies of algorithms on Interval graphs and circular - arc graphs, Ph.D. thesis, S.P. Mahila Visvavidyalayam, India, (1999).

[5] Sampath kumar, E. Neeralagi, P.S. - The neighborhood number of a graph, Indian J. Pure. Appl. Math, 16 (1985), 126-132. 